\title{
Peripheral Latanoprost Administration Lowers Intraocular Pressure in the Wistar Rat
}

\author{
Maciej Świątkiewicz (D) · Marlena Wełniak-Kamińska (D) · \\ Michał Fiedorowicz (D) - Agnieszka Kamińska • Robert Rejdak (D) · \\ Paweł Grieb
}

Received: March 11, 2020 / Published online: May 7, 2020

(c) The Author(s) 2020

\begin{abstract}
Purpose: Instillation of latanoprost eye drops into the conjunctival sac to lower intraocular pressure (IOP) is the most frequently used treatment for primary open-angle glaucoma. The aim of this study was to evaluate the influence of latanoprost on IOP in the rat when applied peripherally.

Methods: A rodent-dedicated tonometer was used to measure IOP in conscious adult male normotensive Wistar rats habituated to the measurement procedure. Commercially available $0.005 \%$ latanoprost solutions were continuously delivered to the periphery of the eye over 7 days using mini-pumps inserted subcutaneously in the animal's back, and IOP was
\end{abstract}

Digital Features To view digital features for this article go to https://doi.org/10.6084/m9.figshare.12180984.

M. Świątkiewicz (₫) · M. Wełniak-Kamińska ·

M. Fiedorowicz · P. Grieb

Department of Experimental Pharmacology,

Mossakowski Medical Research Centre, Polish

Academy of Sciences, Warsaw, Poland

e-mail: mswiatkiewicz@imdik.pan.pl

\section{A. Kamińska}

Faculty of Medicine, Collegium Medicum, Cardinal

Stefan Wyszynski University, Warsaw, Poland

R. Rejdak

Department of General Ophthalmology and

Pediatric Ophthalmology Service, Medical

University of Lublin, Lublin, Poland measured daily. For comparison, a solution containing an equimolar concentration of latanoprost acid, an active compound of latanoprost, was similarly infused into the eyes of different Wistar rats.

Results: Continuous subcutaneous infusion of latanoprost gradually decreased the IOP; the stable nadir of IOP, which was $20 \%$ lower than that prior to the start of infusion, was reached on day 3. The effect was statistically significant and fully reversed 2 days after cessation of drug delivery. Continuous subcutaneous application of the solution containing an equimolar amount of latanoprost acid did not appreciably influence the IOP.

Conclusion: Subcutaneous continuous delivery of latanoprost decreased the IOP in the conscious normotensive Wistar rats in this study. If this effect is confirmed in humans, it may open the possibility of using peripheral systems of drug delivery, which could significantly improve patient compliance.

Keywords: Intraocular pressure; Latanoprost; Latanoprost acid; Peripheral application; Rat 


\section{Key Summary Points}

\section{Why carry out this study?}

Latanoprost-containing eye drops are frequently considered the almost perfect drug for lowering lower intraocular pressure (IOP), but it is associated with poor long-term adherence to treatment, a major drawback.

The aim of this study was to investigate the possibility that continuous subcutaneous infusion of latanoprost decreases IOP.

\section{What was learned from this study?}

Continuous subcutaneous application of latanoprost at a location distant from the eyeball in young adult normotensive Wistar rats achieved a remarkable lowering of IOP.

These results, if confirmed in clinical study, may open the possibility of using peripheral systems of drug delivery, which could significantly improve patient compliance to pharmacological treatment of primary open-angle glaucoma.

\section{INTRODUCTION}

Eye drops containing $50 \mu \mathrm{g} / \mathrm{mL}$ of latanoprost, a lipophilic ester of prostaglandin F2alpha (PGF2 $\alpha$ ), received US and European approval for the treatment of ocular hypertension and glaucoma in 1996 and has subsequently become the most popular first-line pharmacological treatment for glaucoma [1]. Latanoprost is listed in the current World Health Organization Model List of Essential Medicines [2], and eye drops containing this substance as the active principle are widely available, both as the reference medicine (Xalatan; Pfizer) and as numerous generics [1]. 
Latanoprost is a lipophilic ester prodrug exhibiting low pharmacological activity. When latanoprost-containing eye drops are administered to the conjunctival sac, a deposit of this lipophilic compound is thought to be formed in the cornea, followed by gradual hydrolysis (activation) by the corneal esterases. This process is believed to increase the concentration of the active form of the drug, latanoprost acid, in the aqueous humour in the eye anterior chamber and, subsequently, in other ocular tissues [9]. Reduction of the IOP results from the increased outflow of aqueous humour from the anterior chamber of the eye to the aqueous veins, while its production rate remains the same [10].

Studies in healthy human volunteers performed by the manufacturer of the reference drug Xalatan ${ }^{\circledR}$ (Pfizer Inc.) have shown that $77-88 \%$ of latanoprost dose administered to the eye surface is absorbed into the blood [11]. Nonetheless, the authors of one study [12] reported that the plasma half-life of radioactive (tritiated) latanoprost injected intravenously is very short, namely $17 \mathrm{~min}$, due to elimination by the liver. In addition, it has been implicitly assumed that latanoprost absorbed into blood after being administered to the conjunctival sac has no impact on the pharmacodynamic effect of lowering IOP. Consequently, the possibility that latanoprost can reduce IOP when applied systemically has not been investigated.

However, in the case of timolol, another drug routinely applied in the form of eye drops to decrease IOP, administration to one eye of healthy volunteers was associated with a decrease of IOP in both eyes recorded $1 \mathrm{~h}$ and $2 \mathrm{~h}$ later [13]. Further, two independent groups have reported that, in patients suffering from ocular hypertension, sublingual application of the drug was as effective in decreasing IOP over the subsequent $2 \mathrm{~h} \mathrm{[14]}$ and $8 \mathrm{~h} \mathrm{[15]} \mathrm{as} \mathrm{topical}$ treatment. Latanoprost/timolol fixed-combination eye drops, a combination that is distinctly more active than each drug alone, has recently become commercially available [16]. While we were unsuccessful in finding pharmacokinetic data on this drug combination, the results of a recent pharmacokinetics study performed on rabbits using eye drops containing a fixed combination of timolol and another antiglaucoma drug (brimonidine) have been published. In this study, the half-life of timolol in plasma following topical administration was approximately $1 \mathrm{~h}$, similar to that of brimonidine [17].

The aim of the pilot study reported here was to determine whether the continuous subcutaneous infusion of latanoprost decreases IOP. Conscious normotensive Wistar rats habituated to IOP measurements were used as study subjects.

\section{METHODS}

The study was conducted on young adult male Wistar rats (body weight 250-300 g) obtained from the outbred stock maintained at the Animal House of the Mossakowski Medical Research Centre. The animals were kept under standard conditions (12/12-h light/dark cycle, with lights on at 8:00 am GMT +2 ; free access to food and water), in accordance with the requirements of the Directive 2010/63/EU of the European Parliament and of the Council on the protection of animals used for scientific purposes. Animal procedures were approved by the 4th Local Ethics Committee for Animal Experimentation in Warsaw, carried out in accordance with the Association for Research in Vision and Ophthalmology (ARVO) Statement for the Use of Animals in Ophthalmic and Vision Research as well as recommendations in the Guide for the Care and Use of Laboratory Animals of the National Institutes of Health, and documented in compliance with the ARRIVE (Animal Research: Reporting of In Vivo Experiments) guidelines.

IOP measurements were performed in a standardized manner by a researcher blinded to treatment group. Conscious animals were subjected to IOP measurements performed with a TonoLab TV02 rodent-dedicated tonometer (Icare Finland Oy, Vantaa, Finland) according to Pease et al. [18], as described in detail in our previous publication [19]. The IOP measurement at each time point was repeated thrice, and the data were averaged for further analysis. Prior to the experiment, the rats were habituated to the procedure of IOP measurement. 
Each habituation session lasted approximately $10 \mathrm{~min}$ and consisted of immobilizing rat in a customized restrainer and performing the IOP measurement. The sessions were repeated daily for at least 10 days.

Drug formulations used in the experiments were two brands of $0.005 \%$ latanoprost eye drops purchased from a local pharmacy: (1) eye drops containing the benzalkonium chloridecontaining reference drug Xalatan (Pfizer); (2) eye drops containing the benzalkonium-free drug Monoprost (Théa Pharma). For control experiments, an equimolar water solution of latanoprost acid ( $45 \mu \mathrm{g} / \mathrm{mL}$; Everlight Chemical, Taipei, Taiwan) was prepared.

For continuous subcutaneous administration Alzet osmotic pumps (Durect Co., Cupertino, USA; model 2001, lot no. 10348-15) were filled with solutions of latanoprost or latanoprost acid and implanted beneath the skin of the animal's back, according to the manufacturer's instructions (Fig. 1). Briefly, under isoflurane anesthesia a small incision was made on the left side of the body between the scapulae, and a small pocket was formed by spreading the subcutaneous connective tissues with forceps. The osmotic pump was inserted into this pocket with the infusion port directed caudally, and the incision was sutured. The pump, which according to the manufacturer's specification delivers solution continuously for 7 days, was not repositioned or removed before the end of the experiments. IOP measurements were performed at $10 \mathrm{am}$, just before implantation of the Alzet pump, and once daily on subsequent days.

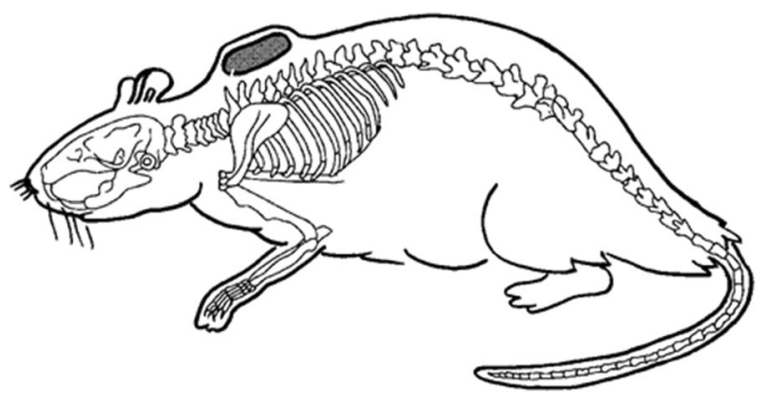

Fig. 1 Localization of Alzet osmotic pump implanted beneath the skin of a rat's back. The osmotic pump was implanted for continuous peripheral administration of latanoprost or latanoprost acid
Data are presented as the mean \pm standard error of the mean. The GraphPad Prism for Windows ver. 6.04 software package (GraphPad Software, San Diego, CA, USA) was used for statistical assessment of the data. Differences between groups were tested using analysis of variance (ANOVA) test or Friedman nonparametric ANOVA and post hoc Dunn's test depending on whether normal distribution of the data was confirmed or not (using D'Agostino-Pearson omnibus and Shapiro-Wilk normality test). Differences between groups were considered insignificant when $p>0.05$.

\section{RESULTS}

Continuous subcutaneous peripheral application of $0.005 \%$ latanoprost solution for 1 week gradually decreased IOP, reaching the stable nadir of almost $20 \%$ lower IOP values on day 3 of application. No appreciable difference was noted in decreases in IOP between eyes receiving the benzalkonium chloride-containing solution ( $0.005 \%$ latanoprost; Xalatan) and those receiving the benzalkonium-free latanoprost solution (0.005\% latanoprost; Monoprost)

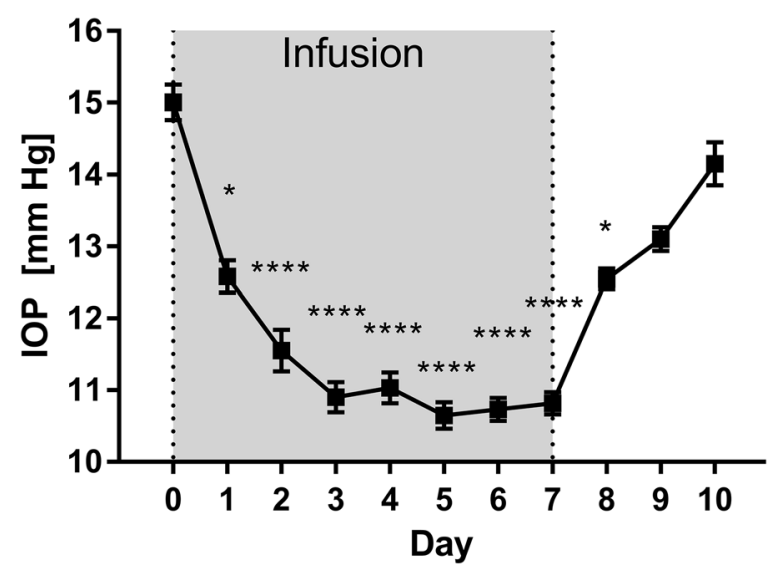

Fig. 2 Intraocular pressure (IOP) following continuous subcutaneous administration of $0.005 \%$ latanoprost solution. Number of eyes $n=20$ (10 animals). Asterisks indicate statistical significance of changes due to treatment compared to the control measurement on day 0 (just before pump implantation): ${ }^{*} p \leq 0.05,{ }^{* * * *} p \leq 0.0001$. Data were analysed using Friedman nonparametric analysis of variance and the post-hoc Dunn's test 


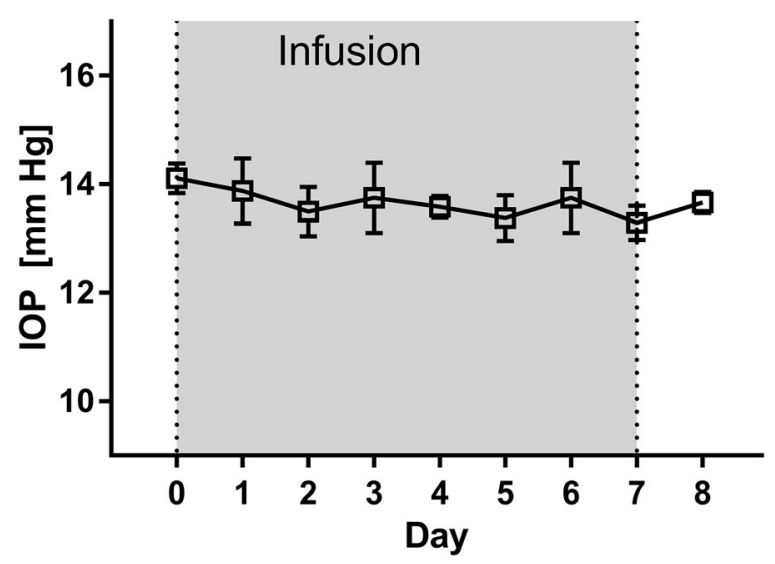

Fig. 3 IOP following continuous subcutaneous administration of latanoprost acid (control experiment). Number of eyes $n=8$ ( 4 animals)

( $n=4$ and $n=16$, respectively), and all data were averaged. The effect of treatment was highly statistically significant and fully reversed 2 days after cessation of drug delivery (Fig. 2).

The continuous subcutaneous application of latanoprost acid at a dose equimolar to that used in the experiments did not produce any change in the IOP (Fig. 3).

\section{DISCUSSION}

Latanoprost-containing eye drops to be applied daily are the most popular IOP-reducing treatment for patients with ocular hypertension or glaucoma [20], but poor patient compliance is the main disadvantage of this type of drug formulation. A system for the sustained topical delivery of the PGF2 $\alpha$ ester drug bimatoprost from biodegradable polymer inserted intracamerally has recently entered the clinical development phase [21], being recognized as a potentially favorable alternative to eye drops applied every day. Other systems and devices proposed to date for decreasing IOP by sustained drug delivery also involve topical application, such as drug-eluting contact lenses, intraocular implants, punctal plugs, among others (reviewed in a previous publication [22]). The common disavantage of these solutions is the requirement for implantation or some other type of manipulation in the very sensitive area of eyeball and its immediate surroundings.

The majority of researchers believe that latanoprost, because of its lipophilic nature, sinks into the cornea following instillation into the conjunctival sac, where it is readily hydrolysed by corneal esterases. The product of enzymatic hydrolysis, water-soluble latanoprost acid, is then released into the aqueous humour in the anterior chamber of the eye, reaching the other ocular tissues (e.g. iris and ciliary body) from, so to say, "inside the eye".

The results of our study indicate that a notable lowering of IOP was achieved in our normotensive Wistar rats by the continuous subcutaneous application of latanoprost at a location distant from the eyeball. At first glance this effect would seem to be unexpected and surprising. However, the authors of one pharmacokinetics study instilled tritiated latanoprost onto the eye surface of rabbits and found that the radioactivity, which indeed was the highest in the cornea, seemed to appear instantaneously also in other parts of the eye (iris and ciliary body), and was initially slightly but visibly delayed in the aqueous fluid (see Fig. 4 in Ref. [22]). These data would appear to contradict the notion that to decrease IOP latanoprost must be first hydrolysed by esterases in the cornea followed by its water-soluble active form reaching prostaglandin receptors in the eye by diffusion or convection through the aqueous fluid. Our data are more compatible with the concept that latanoprost somehow reaches the site of its pharmacological action via systemic absorption.

A mechanism that enables latanoprost infused subcutaneously at a very slow rate to cause lowering IOP in a normotensive Wistar rat remains undetermined, but some conjectures may be put forward. In our experiments, the equimolar amount of water-soluble latanoprost acid, the active form of latanoprost, infused subcutaneously at the same rate (i.e. the control experiments) did not appreciably influence IOP. This indicates that hydrolysis of latanoprost at the infusion site either does not occur or, more probably, is only partial, thereby allowing the unhydrolysed lipophilic ester prodrug to escape elimination by the liver and be transported to 


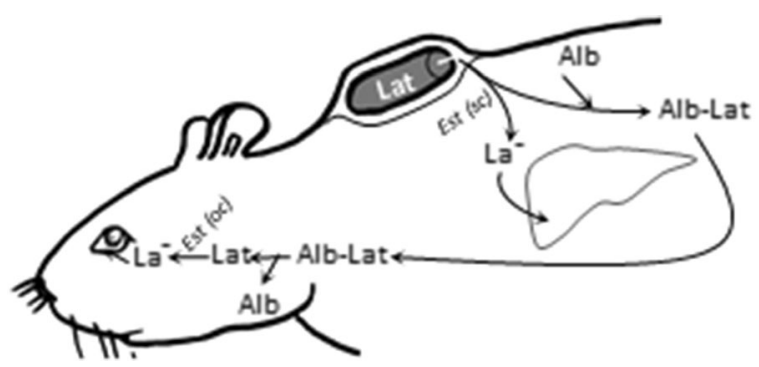

Fig. 4 A hypothetical mechanism for the decrease in IOP following continuous subcutaneous infusion of latanoprost $(L A T)$ at a location distant from the eye. Infused LAT is subjected to two competing reactions: (1) hydrolysis to latanoprost acid $\left(L A^{-}\right)$, catalysed by esterases located subcutaneously (Est. sc.), and (2) binding to blood plasma proteins, such as albumin. $\mathrm{LA}^{-}$is cleared from the blood by the liver and does not reach the eye. Albumin-bound latanoprost $(A l b-L A T)$ travels to the eye in the blood and is locally hydrolysed by esterases in the ocular tissues (Est. $o c$.)

sites of its pharmacological action through the blood. Most likely, under slow subcutaneous infusion, latanoprost binds to plasma proteins, such as albumin, which considerably affects its pharmacokinetics, as happens with many other drugs $[23,24]$. Of note, the IOP-lowering effect of the slow subcutaneous infusion of latanoprost reached its maximum only on the third day of uninterrupted infusion. It is possible that a long time is required to equilibrate latanoprst binding to plasma proteins, which may be a precondition for delivering a pharmacologically effective amount of this drug to the eye. This hypothesis is explained in Fig. 4.

\section{CONCLUSION}

The rat eye and human eye share many important biological similarities and, consequently, rat models are widely used for studies of various aspects of glaucoma [25-27]. We conducted our experiments on rats with normal IOP; thus, it may be argued that we should have used one of the rat models of glaucoma. In human glaucoma patients the magnitude of the IOP-lowering effect of latanoprost in hypertensive eyes is proportional to the degree of hypertension [12]. Although we cannot guarantee that IOP reduction caused by continuous peripheral subcutaneous infusion of latanoprost observed in normotensive rats will occur in humans, we suspect that the hypotensive effect will also be evident in rats with ocular hypertension. However, further investigation of this phenomenon is warranted only if it also occurs in humans. If our results are confirmed in human study, the possibility of using peripheral systems of drug delivery would be increased, which could significantly improve patient compliance to pharmacological treatment of primary open-angle glaucoma.

\section{ACKNOWLEDGEMENTS}

Funding. The study was supported by GBJ Pharma, Ltd., Warsaw, Poland. The Rapid Service Fee was funded by the Mossakowski Medical Research Centre, Polish Academy of Sciences.

Authorship. All named authors meet the International Committee of Medical Journal Editors (ICMJE) criteria for authorship for this article, take responsibility for the integrity of the work as a whole, and have given their approval for this version to be published.

Disclosures. Maciej Świątkiewicz, Marlena Wełniak-Kamińska, Michał Fiedorowicz and Paweł Grieb are holders of patent application related to systems for continuous peripheral administration of latanoprost to reduce intraocular pressure, assigned to GBJ Pharma, Ltd., Warsaw, Poland, and Mossakowski Medical Research Centre, Polish Academy of Sciences, Warsaw, Poland. Agnieszka Kamińska and Robert Rejdak have nothing to declare.

Compliance with Ethics Guidelines. Animal procedures were approved by the 4th Local Ethics Committee for Animal Experimentation in Warsaw, carried out in accordance with the Association for Research in Vision and Ophthalmology (ARVO) Statement for the Use of Animals in Ophthalmic and Vision Research as well as recommendations in the Guide for the 
Care and Use of Laboratory Animals of the National Institutes of Health, and documented in compliance with the ARRIVE (Animal Research: Reporting of In Vivo Experiments) guidelines.

Data Availability. The datasets obtained and analysed during the current study are available from the corresponding author on reasonable request.

Open Access. This article is licensed under a Creative Commons Attribution-NonCommercial 4.0 International License, which permits any non-commercial use, sharing, adaptation, distribution and reproduction in any medium or format, as long as you give appropriate credit to the original author(s) and the source, provide a link to the Creative Commons licence, and indicate if changes were made. The images or other third party material in this article are included in the article's Creative Commons licence, unless indicated otherwise in a credit line to the material. If material is not included in the article's Creative Commons licence and your intended use is not permitted by statutory regulation or exceeds the permitted use, you will need to obtain permission directly from the copyright holder. To view a copy of this licence, visit http://creativecommons.org/licenses/by$\mathrm{nc} / 4.0 /$.

\section{REFERENCES}

1. Digiuni M, Fogagnolo P, Rossetti L. A review of the use of latanoprost for glaucoma since its launch. Expert Opin Pharmacother. 2012;13:723-45.

2. WHO Essential Medicines and Health Products Department. World Health Organization model list of essential medicines, 21st edn. World Health Organization. 2019. https://www.who.int/ medicines/publications/essentialmedicines/en/. Accessed 10 Mar 2020.

3. Klimko PG, Sharif NA. Discovery, characterization and clinical utility of prostaglandin agonists for the treatment of glaucoma. Br J Pharmacol. 2019;176: 1051-8.
4. Pfizer Inc./Pfizer Ltd. Pfizer material safety data sheet. Latanoprost solution. Pfizer Inc. 2009. https://www.pfizer.com/sites/default/files/products/ material_safety_data/LATANOPROST\%20SOLUTION. pdf. Accessed 08 Mar 2020.

5. Olthoff CM, Schouten JS, van de Borne BW, Webers CA. Noncompliance with ocular hypotensive treatment in patients with glaucoma or ocular hypertension an evidence-based review. Ophthalmology. 2005;112:953-61.

6. Belhassen M, Laforest L, Licaj I, Van Ganse E. Early adherence to anti-glaucoma therapy: an observational study. Therapie. 2016;71:491-9.

7. Zarnowski T, Wyszynska A, Rekas M. Comparison of self-reported and objective adherence to antiglaucoma medications. J Ocul Pharmacol Ther. 2016;32:403-4.

8. Yeaw J, Benner JS, Walt JG, Sian S, Smith DB. Comparing adherence and persistence across 6 chronic medication classes. J Manag Care Pharm. 2009;15:728-40.

9. Sjoquist B, Basu S, Byding P, Bergh K, Stjernschantz $\mathrm{J}$. The pharmacokinetics of a new antiglaucoma drug, latanoprost, in the rabbit. Drug Metab Dispos. 1998;26:745-54.

10. Dinslage S, Hueber A, Diestelhorst M, Krieglstein G. The influence of latanoprost $0.005 \%$ on aqueous humor flow and outflow facility in glaucoma patients: a double-masked placebo-controlled clinical study. Graefes Arch Clin Exp Ophthalmol. 2004;242:654-60.

11. Sandoz Canada Inc. Product monograph. PrSandoz latanoprost. Latanoprost ophthalmic solution, 50 mcg/mL. Sandoz Canada Inc. 2017. https://www. sandoz.ca/sites/www.sandoz.ca/files/Sandoz\%20Lat anoprost\%20Product\%20Monograph.pdf. Accessed 05 Mar 2020.

12. Sjoquist B, Stjernschantz J. Ocular and systemic pharmacokinetics of latanoprost in humans. Surv Ophthalmol. 2002;47(Suppl 1):S6-12.

13. Dunham CN, Spaide RF, Dunham G. The contralateral reduction of intraocular pressure by timolol. Br J Ophthalmol. 1994;78:38-40.

14. Sadiq SA, Vernon SA. Sublingual timolol-an alternative to topical medication in glaucoma? $\mathrm{Br} \mathrm{J}$ Ophthalmol. 1996;80:532-5.

15. Quaranta L, Pasquini L, Cassamali M, Hauranieh N, Gandolfo E, Quaranta CA. Ocular hypotensive effect of sublingual administration of timolol. Int Ophthalmol. 1996;20:49-51. 
16. Konstas AG, Mocan MC, Katsanos A, Voudouragkaki IC, Irkec M. Latanoprost/timolol fixed combination for the treatment of glaucoma. Expert Opin Pharmacother. 2013;14:1815-27.

17. Suzuki G, Kunikane E, Shinno K, Kozai S, Kurata M, Kawamura A. Ocular and systemic pharmacokinetics of brimonidine and timolol after topical administration in rabbits: comparison between fixed-combination and single drugs. Ophthalmol Ther. 2020;9:115-25.

18. Pease ME, Cone FE, Gelman S, Son JL, Quigley HA. Calibration of the TonoLab tonometer in mice with spontaneous or experimental glaucoma. Investig Ophthalmol Vis Sci. 2011;52:858-64.

19. Fiedorowicz M, Orzel J, Kossowski B, et al. Anterograde transport in axons of the retinal ganglion cells and its relationship to the intraocular pressure during aging in mice with hereditary pigmentary glaucoma. Curr Eye Res. 2018;43:539-46.

20. Aspberg J, Heijl A, Johannesson G, Linden C, Andersson-Geimer S, Bengtsson B. Intraocular pressure lowering effect of latanoprost as first-line treatment for glaucoma. J Glaucoma. 2018;27: 976-80.
21. Lewis RA, Christie WC, Day DG, et al. Bimatoprost sustained-release implants for glaucoma therapy: 6-month results from a phase I/II clinical trial. Am J Ophthalmol. 2017;175:137-47.

22. Aref AA. Sustained drug delivery for glaucoma: current data and future trends. Curr Opin Ophthalmol. 2017;28:169-74.

23. Kratz F. Albumin as a drug carrier: design of prodrugs, drug conjugates and nanoparticles. J Control Release. 2008;132:171-83.

24. Zhivkova ZD. Studies on drug-human serum albumin binding: the current state of the matter. Curr Pharm Des. 2015;21:1817-30.

25. Pang IH, Clark AF. Rodent models for glaucoma retinopathy and optic neuropathy. J Glaucoma. 2007; 16:483-505.

26. Chen S, Zhang X. The rodent model of glaucoma and its implications. Asia Pac J Ophthalmol. 2015;4: 236-41.

27. Morrison JC, Johnson E, Cepurna WO. Rat models for glaucoma research. Prog Brain Res. 2008;173: 285-301. 